they presented with a coexisting Axis I condition that might have a major impact on their ability to effectively participate in the groups, such as severe social phobia or obsessive-compulsive disorder.

Second, regarding details of status and/or type of Axis I/II comorbidities, we would like to point out that this was already covered for the 2-year follow-up in a previous paper. ${ }^{1}$

Third, we defined recurrence both based on severity ratings and DSM-IV criteria; these are narrow criteria which are much more reliable than just asking for diagnostic criteria alone or rating scale scores. We disregarded the possibility of using a lifechart method to catch subsyndromal fluctuations because this method has not shown good reliability and would likely capture a lot of noise.

Fourth, criteria for hospitalisation were those used at the Barcelona Bipolar Disorders Program: any patient presenting an episode that, owing to its severity, cannot be managed in an out-patient setting and/or any patient presenting suicide risk or representing a risk for third persons.

Fifth, as clearly explained in our manuscript, the primary outcome of the trial was time to recurrence. Secondary outcomes included time spent ill and number of recurrences. Our original submission included a full data report on those secondary variables, which had to be condensed owing to space constraints. The analysis of the number of recurrences was, as explained in the Method, performed by means of ANCOVA and therefore the mean values for each group are just orientive.

Finally, we acknowledge a typing error in Table 2 referring to the number of days spent in depression. The right values should be: control group, mean $=398.55$ days $($ s.d. $=364.16)$; psychoeducation group, mean $=93.28$ days $($ s.d. $=165.46)$. The standard deviation for the control group was mistakenly repeated replacing the mean number of days spent in depression for the psychoeducation group. After correcting this error, data regarding mean number of days spent in each episode tally with the total duration for both groups. As this was only a typing error, it does not change any statistics. We have been informed of this mistake by other readers and have already proceeded to issue the corresponding erratum.

1 Colom F, Vieta E, Sánchez-Moreno J, Martínez-Arán A, Torrent C, Reinares M, et al. Psychoeducation in bipolar patients with comorbid personality disorders. Bipolar Disord 2004; 6: 294-8.

Francesc Colom, Bipolar Disorders Program, Hospital Clinic of Barcelona, Villarroel 170, 08036 Barcelona, Spain. Email: fcolom@clinic.ub.es; Eduard Vieta, Bipolar Disorders Program, Institute of Neurosciences, Hospital Clinic, IDIBAPS, CIBER-SAM, University of Barcelona, Spain

doi: 10.1192/bjp.195.2.180a

\section{Abortion and mental health: established facts reconsidered}

Tyrer's 'From the Editor's desk' lyrically asserted that in relation to the paper by Fergusson et $a l^{1}$ and other studies, 'In the parched desert of ignorance and prejudice every established fact becomes an oasis. By "established fact" I mean one that defines the field, the one that all the related and restlessly inchoate facts gather round and say "I belong here", and then fall into line behind it."

Fergusson $e a^{1}$ conclude that there is evidence that abortion may be associated with a small increase in risk of mental disorders and in comparison, other pregnancy outcomes were not associated with increased risk. Although we acknowledge that aspects of their analytic design are strong and carefully implemented, we believe that the analyses have not maximised the potential of the dataset and that therefore, your editor's rhetorical confidence is not yet justified. We advance the following reasons.
First, Fergusson et al dichotomised each pregnancy exposure. Of 534 women in the Christchurch cohort, 284 had had pregnancies. Women making decisions about terminating pregnancies may have prior pregnancy events and potentially cumulative losses will have different mental health impacts compared with termination as the outcome of a first pregnancy. Pregnancy variables are not independent and mutual adjustment in models for other outcomes will not account for the interactions between pregnancy outcomes. A more useful analysis would have been with a composite variable with never having had a pregnancy event as the reference category.

Second, the combining of therapeutic abortion for fetal malformation with abortion by choice is inappropriate. Most abortions are first trimester. There is an argument for separating termination of pregnancy by gestational age, so that the mental health impact of those in the second or third trimester are visible and separate. It is possible that terminating a wanted pregnancy because of fetal abnormality would be more distressing than an early unwanted pregnancy.

Third, many authors (including Fergusson et al) have found strong relationships between intimate partner violence and poor mental health, and between intimate partner violence and increased association with reporting terminations. ${ }^{3-6}$ Despite the potential to include the much more rigorous measure from their previous study of partner violence among this cohort, the authors have excluded their strongest measures of partner violence in this analysis, leaving a major covariate poorly measured.

Fergusson et al conclude that the evidence for abortion impact is small but clear - even causal. Yet there is no evidence that the risks associated with other pregnancy outcomes, particularly loss, are different from those estimated for abortion (see Charles et $\mathrm{l}^{7}$ ), nor that mental health disorders are incident after an abortion. This could have been statistically tested using logistic regression among the range of statistical tests already carried out.

It is a pity that such a good cohort study has not been better analysed. With the above adjustments, the authors would be better placed to more clearly identify the vulnerable groups they are wisely seeking to identify.

1 Fergusson DM, Horwood LJ, Boden JM. Abortion and mental health disorders: evidence from a 30-year longitudinal study. Br J Psychiatry 2008; 193: 444-51.

2 Tyrer P. From the Editor's desk. Br J Psychiatry 2008; 193: 524.

3 Taft AJ, Watson LF. Depression and termination of pregnancy (induced abortion) in a national cohort of young Australian women: the confounding effect of women's experience of violence. BMC Public Health 2008; 8: 75.

4 Fergusson DM, Horwood L, Ridder EM. Partner violence and mental health outcomes in a New Zealand birth cohort. J Marriage Fam 2005; 67: 1103-19.

5 Hegarty KL, Gunn J, Chondros P, Small R. Association between depression and abuse by partners of women attending general practice: descriptive, cross-sectional survey. BMJ 2004; 328: 621-4.

6 Gazmararian JA, Adams MM, Saltzman LE, Johnson CH, Bruce FC, Marks JS, et al. The relationship between pregnancy intendedness and physical violence in mothers of newborns. The PRAMS Working Group. Obstet Gynecol 1995; 85: 1031-8.

7 Charles VE, Polis CB, Sridhara SK, Blum RW. Abortion and long term mental health outcomes: a systematic review of the evidence. Contraception 2008; 78: $436-50$.

Angela J. Taft, Mother and Child Health Research, La Trobe University, 324-328 Little Lonsdale Street, Melbourne, Austrlia. Email: a.taft@latrobe.edu.au; Lyndsey Watson, Mother and Child Health Research, La Trobe University, Australia

doi: 10.1192/bjp.195.2.181

Authors' reply: Taft \& Watson claim that we measured pregnancy history using dichotomous measures and that this fails to represent the complexities of pregnancy history. This claim misrepresents our analysis. 